\title{
Scaling Solutions and Reconstruction of Scalar Field Potentials
}

\author{
Claudio Rubano ${ }^{1}$ and John D. Barrow ${ }^{2}$ \\ ${ }^{1}$ Dept. of Phys. Sciences - Univ. Federico II and INFN Sez. di Napoli \\ Complesso Universitario di Monte S. Angelo, \\ via Cintia, Ed. G, I-80126 Napoli - Italy \\ ${ }^{2}$ DAMTP, Centre for Mathematical Sciences, Cambridge University, \\ WilberforceRd., Cambridge CB3 0WA, U.K.
}

(October 27, 2018)

\begin{abstract}
Starting from the hypothesis of scaling solutions, the general exact form of the scalar field potential is found. In the case of two fluids, it turns out to be a negative power of hyperbolic sine. In the case of three fluids the analytic form is not found, but is obtained by quadratures.
\end{abstract}

PACS: $98.80 . \mathrm{Cq}, 98.80 . \mathrm{Hw}, 04.20 . \mathrm{Jb}$

\section{Introduction}

In cosmological theories containing scalar fields with a self-interaction potential $V(\varphi)$, it is sometimes possible to reconstruct the required scalar field potential for a simple cosmological solution. In the context of inflationary theory, this approach was used by various authors [1], [2], [3], [4], [5], [6] [7] [8] [9], [10], and was primarily interested in the behaviour of solutions containing only scalar fields undergoing inflation. The more recent invocation of a scalar field as a dark matter source responsible for accelerating the universe today, under the pseudonym of "quintessence", [11], [12], [13], [14], [15], is mathematically almost identical, but places different requirements on the solutions. In particular, it is of interest to find solutions which contain both perfect fluids and scalar fields. In this new context, interesting applications of the reconstruction approach were made by Chiba and Nakamura [16] and Saini et al. [17.

In this paper, we seek exact cosmological solutions for a universe containing a perfect fluid and a scalar field. We start from the assumption that the energy density of the scalar field scales as an exact power of the scale factor: $\rho_{\varphi}=K a^{-n}$, which is equivalent to imposing an equation of state linking the pressure and density, of the form $p_{\varphi}=w \rho_{\varphi}$ with constant $w=n / 3-1$. For a flat Friedmann universe, it is then possible to find an explicit exact form of the potential in terms of $n, H_{0}$, and $\Omega_{m 0}$. The form of the resulting general solution has instructive features which will be discussed below. 


\section{Derivation of the Potential}

Consider a cosmological fluid with two non-interacting components: perfect-fluid matter and a scalar field $\varphi$ with potential $V(\varphi)$. In the flat universe case, we have the equations

$$
\begin{gathered}
3 H^{2}=\mathcal{G}\left(\rho_{m}+\rho_{\varphi}\right) \\
\ddot{\varphi}+3 H \dot{\varphi}+V^{\prime}(\varphi)=0 \\
\rho_{\varphi}=\frac{1}{2} \dot{\varphi}^{2}+V(\varphi) \\
\rho_{m}=D a^{-m},
\end{gathered}
$$

where $H=\dot{a} / a$, where $a(t)$ is the expansion scale factor is the Hubble expansion rate, and overdot denotes differentiation with respect to the comoving proper time $t ; \mathcal{G}=8 \pi G / c^{2}$ and $V^{\prime}=d V / d \varphi$. The constant $m$ depends on the type of perfect fluid present.

We normalize the present value of the scale factor to $a_{0}=1$, without loss of generality, and for brevity denote the present matter-density parameter, $\Omega_{m 0}$, by $\Omega_{0}$, and define

$$
D=3 H_{0}^{2} \Omega_{0} / \mathcal{G}
$$

If we make the assumption that

$$
\rho_{\varphi}=K a^{-n}
$$

with $n<m$, so the scalar field can dominate at late times, and define

$$
K=3 H_{0}^{2}\left(1-\Omega_{0}\right) / \mathcal{G}
$$

then from Eqs. (2), (3) and (6) we obtain

$$
\dot{\varphi}^{2}=\frac{K n}{3} a^{-n}
$$

Since

$$
\frac{d \varphi}{d t}=H a \frac{d \varphi}{d a}
$$

we have

$$
\left(\frac{d \varphi}{d a}\right)^{2}=\frac{K}{3 H_{0}^{2}} \frac{\sqrt{n}}{\Omega_{0} a^{n-m+2}+\left(1-\Omega_{0}\right) a^{2}}
$$

which gives

$$
\begin{aligned}
\varphi(a)=\int \sqrt{\frac{1-\Omega_{0}}{\mathcal{G}}} \frac{\sqrt{n} d a}{\sqrt{\Omega_{0} a^{n-m+2}+\left(1-\Omega_{0}\right) a^{2}}} & \\
& =\frac{2 \sqrt{n}}{\sqrt{\mathcal{G}}(m-n)} \operatorname{arcsinh}\left(\sqrt{\frac{1-\Omega_{0}}{\Omega_{0}}} a^{\frac{m-n}{2}}\right)+\varphi_{i n} .
\end{aligned}
$$


Returning to the potential, we get from Eqs. (3) and (9)

$$
V=K a^{-n}-\frac{1}{2} \dot{\varphi}^{2}=\frac{3 H_{0}^{2}}{\mathcal{G}}\left(1-\Omega_{0}\right)\left(1-\frac{n}{6}\right) a^{-n} .
$$

The scale factor can be easily eliminated, giving eventually

$$
\begin{aligned}
V(\varphi)=\frac{3 H_{0}^{2}}{\mathcal{G}}\left(1-\Omega_{0}\right)\left(1-\frac{n}{6}\right)\left(\frac{1-\Omega_{0}}{\Omega_{0}}\right)^{\frac{n}{m-n}} & \\
& \times\left(\sinh \left(\sqrt{\mathcal{G}} \frac{m-n}{\sqrt{n}}\left(\varphi-\varphi_{i n}\right)\right)\right)^{-\frac{2 n}{m-n}} .
\end{aligned}
$$

This expression, in the case of dust $(m=3)$, coincides with the one presented by 18 (which is in turn a particular case of the treatment of [19]) as well as with the one by 20]. A general discussion about exact solutions for Friedmann equations, which includes ours as a particular case, can be found also in [7], [8], [6]. In [18] and [19], one can find explicit solutions for $a(t)$ and $\varphi(t)$, as well as an extensive discussion. We remark only that this form of potential is good for a tracker solution [13], [14]. Indeed, straightforward computation of the function $\Gamma=V^{\prime \prime} V /\left(V^{\prime}\right)^{2}$, introduced and discussed in these papers, gives

$$
\Gamma=1+\frac{m-n}{2 n}\left(\operatorname{sech}\left(\frac{m-n}{\sqrt{n}} \varphi\right)\right)^{2}>1
$$

as required by the tracking condition.

Our derivation differs from [18], [19] and [20] because it is simpler and is generalised to include all perfect fluid equations of state (other than the $n=6$ case which would correspond to a pure scalar field with no potential). Moreover, it proves that this form of $V(\varphi)$ is the unique solution, if condition (6) is imposed.

\section{Discussion}

The first interesting feature of Eq. (13) is that the slope (as well as the amplitude) of $V(\varphi)$ depends on $n$. This means that it is impossible to obtain a scaling solution, with the same potential slope, when passing from a radiation-dominated $(m=4)$ epoch to a matterdominated $(m=3)$ epoch. Even if we assume that $n$ changes in such a way that the slope remains constant, the coefficient $(1-n / 6)$ changes. Moreover, the effective equation of state of the scalar field also changes and there is no physical mechanism for it to be influenced in this way by the dominating type of matter. Although this situation seems to be unphysical, it is merely an artifact of having sought a solution containing a single perfect fluid. The full solution must be found by including dust, radiation and scalar field from the outset, not by joining the radiation + scalar solution to the dust + scalar solution. If this is done for the

case of dust + radiation + scalar field, with the same arguments as before, it is easy to derive

$$
\begin{aligned}
\varphi & =\int \sqrt{\frac{\Omega_{\varphi}}{\mathcal{G}}} \frac{\sqrt{n} d a}{\sqrt{\Omega_{r} a^{n-2}+\Omega_{d} a^{n-1}+\Omega_{\varphi} a^{2}}}+\varphi_{i n} \\
V & =\frac{3 H_{0}^{2}}{\mathcal{G}} \Omega_{\varphi}\left(1-\frac{n}{6}\right) a^{-n},
\end{aligned}
$$


where $\Omega_{r}, \Omega_{d}$ and $\Omega_{\varphi}$ are the present values of the radiation, dust and scalar field density parameters, respectively, so $\Omega_{r}+\Omega_{d}+\Omega_{\varphi}=1$. These equations give a parametric representation of $V(\varphi)$, which cannot be solved analytically with simple functions, but is otherwise perfectly well defined and interpolates between the dust and radiation solutions of Eq. (13). In our universe today, $\Omega_{r}<<\Omega_{d}$, but at early times the radiation term $\Omega_{r} a^{n-2}$ dominates the dust and cannot be dropped.

A numerical example illustrates the situation: let us set $n=1, \Omega_{\varphi}=0.7, \Omega_{r}=0.0001$, $\Omega_{d}=0.3-\Omega_{r}, 3 H_{0}^{2}=1, \mathcal{G}=1$. Fig. 1 shows that on the first part the "true" potential fits well with Eq. (13) and $m=4$, while Fig. 2 shows that in the late regime the fit should be done with $m=3$.

The simple and attractive form of Eq. (13) is lost, although it might be recovered by a suitable choice of the exponent: via some weighted mean with of $\Omega_{r}$ and $\Omega_{d}$. But there is no way of doing this other than a fit of the numerical values. Moreover, there is no reason why the exponent should depend on the particular values of $\Omega_{r}$ and $\Omega_{d}$. Also, since in this case the scaling feature of the solution is only approximate, the tracker behavior could be affected.

Simple numerical evaluations (with the parameters within the allowed range) show that the "true" potential is very well approximated by

$$
V=\frac{\alpha}{\varphi^{\beta}}-\gamma,
$$

with $\alpha, \beta, \gamma$ positive constants depending on the parameters; in particular, $\beta \approx 2 n /(m-n)$. The negative additive term, $\gamma$, is an artifact of the approximation over a finite range of $\varphi$. This result, already found by [18] for the dust case, shows that the inverse-power potential is effectively equivalent to Eq. (13), so that it is possible to apply to this situation all the known results about tracker solutions. On this point, it is interesting to note that this approximation is very good over the whole range of $a$ from zero to the present-day value ( $a_{0}=1$ according to our normalization). The asymptotic exponential behavior of the hyperbolic potential is therefore important only in the very far future and does not affect the dominance of scalar field now or its behaviour in the recent past.

As a final remark, we should stress that our arguments are based on the arbitrary assumption of Eq. (6). We have shown elsewhere [21] that other forms of exponential potential are perfectly able to reproduce observational data, but of course in these cases $w$ is not constant. It is interesting also to note that, in one of the cases treated in that paper, $w$ is almost perfectly constant, and yet the form of the potential is substantially different from that of Eq. (13). 


\section{REFERENCES}

1 G.F.R. Ellis and M.S. Madsen, Class. Quantum Grav. 8, 667 (1991)

2 J.D. Barrow, Phys. Rev. D 48, 1585 (1993)

3 G. Mangano, G. Miele, C. Stornaiolo, Mod. Phys. Lett. A10 1977 (1995)

4 R. de Ritis et al., Il Nuovo Cimento 109, 403 (1994)

5 J.D. Barrow and P. Parsons, Phys. Rev. D 52, 5576 (1995)

$6 \quad$ R.T. Jantzen and C. Uggla Gen. Rel. Grav. 24, 59 (1991)

7 R.T. Jantzen, C. Uggla and K. Rosquist, Gen. Rel. Grav. 24, 409 (1992)

8 C. Uggla, R.T. Jantzen and K. Rosquist, Phys. Rev., D 51, 5522 (1995)

$9 \quad$ S. Capozziello et al., Il Nuovo Cimento 111, 623 (1996)

10 S. Capozziello et al., In "Proc. XI It. Conf. on Gen. Rel. and Grav. Phys.", Trieste (Italy), Sept. 1994, p. 291, World Scientific, Singapore,1996

11 J.P. Ostriker and P.J. Steinhardt, Nature 377, 600 (1995)

12 R.R. Caldwell, R. Dave, and P.J. Steinhardt, Phys. Rev. Lett. 80, 1582 (1998)

13 I. Zlatev, L. Wang, and P.J. Steinhardt, Phys. Rev. Lett. 82, 896 (1999)

14 P. J. Steinhardt, L. Wang, and I. Zlatev, Phys. Rev. D 59, 123504 (1999)

15 J.D. Barrow, R. Bean \& J. Magueijo, Mon. Not. Roy. Astron. Soc. 316, L41 (2000)

16 T. Chiba and T. Nakamura, Phys. Rev. D 62, 121301 (2000)

17 T.D. Saini et al., Phys. Rev. Lett. 85, 1162 (2000)

18 L.A. Ureña-López and T. Matos, Phys. Rev. D 62, 081302 (2000); T. Matos and L.A. Ureña-López, Class.Quant.Grav. 17 (2000) L75-L81

19 L.P. Chimento and A.S. Jakubi, Int. J. Mod. Phys. D 5, 71 (1995)

20 V. Sahni and A. Starobinsky, Int. J. Mod. Phys. D 9, 373 (2000)

21 C. Rubano and P. Scudellaro, astro-ph/0103335

\section{Figure Captions}

Figure 1: Early-time regime: the dashed curve is the $\mathrm{n}=1$ scalar field potential with dust $(\mathrm{m}=3)$; the solid curve is the $\mathrm{n}=1$ scalar field potential with radiation $(\mathrm{m}=4)$ and lies closer to the points plotting the full numerical solution for dust plus radiation and $\mathrm{n}=$ 1 scalar field potential.

Figure 2: Late-time regime: the dashed curve is the $\mathrm{n}=1$ scalar field potential with radiation $(\mathrm{m}=4)$; the solid curve is the $\mathrm{n}=1$ scalar field potential with dust $(\mathrm{m}=3)$ and lies closer to the points plotting the full solution for dust plus radiation and $n=1$ scalar field potential. 


$$
\text { Fig. } 1
$$

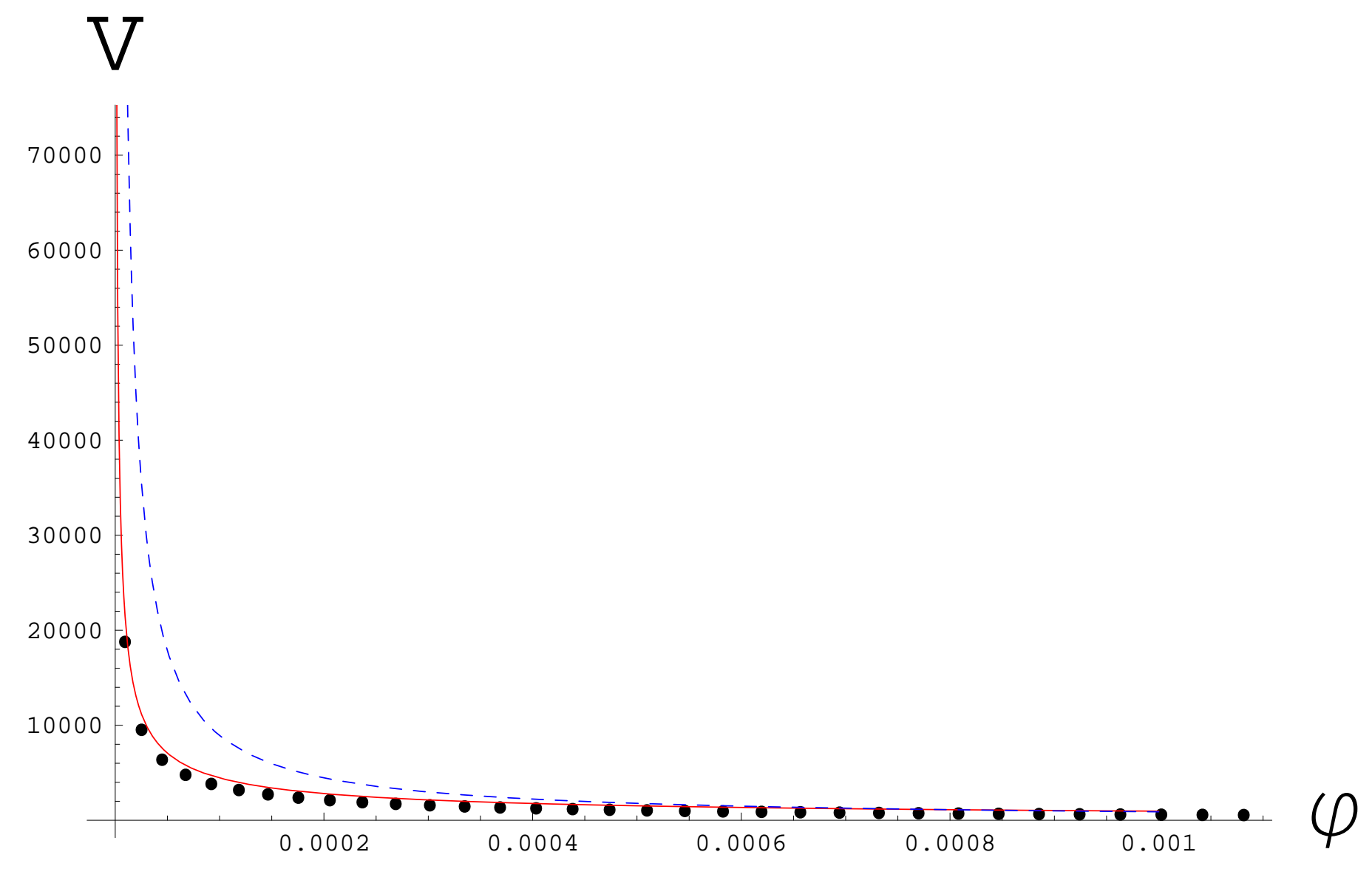


Fig. 2

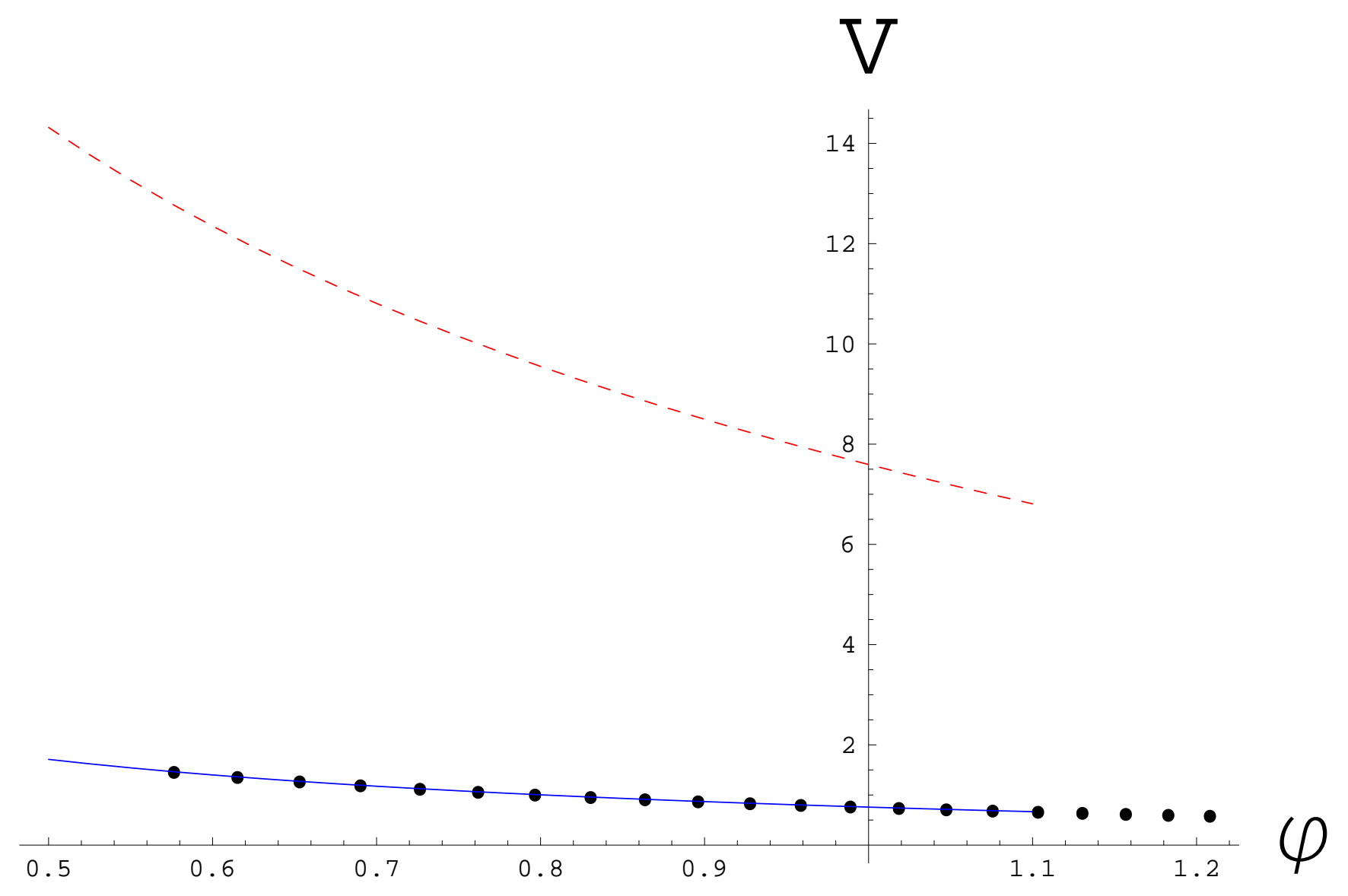

